\title{
Profil Des Infections Ostéoarticulaires En Consultation Rhumatologique Au CHU- Kara (Togo)
}

\author{
Prénam Houzou \\ Service de Rhumatologie, CHU Kara, Université de Kara-Togo, \\ Kodjo Kakpovi
}

Service de Rhumatologie, CHR Lomé Commune, Université de Lomé-Togo,

Eyram Fianyo

Service de Rhumatologie, CHU SylvanusOlympio,

Université de Lomé-Togo,

Viwalé Etonam S. Koffi-Tessio

Service de Rhumatologie, CHR Lomé Commune, Université de Lomé-Togo, Komi Cyrille Tagbor

Service de Rhumatologie, CHU SylvanusOlympio,

Université de Lomé-Togo,

Dadja Essoya Landoh

Organisation Mondiale de la Santé, Lomé, Togo

Owonayo Oniankitan

Service de Rhumatologie, CHU SylvanusOlympio,

Université de Lomé-Togo

\section{Moustafa Mijiyawa}

Service de Rhumatologie, CHR Lomé Commune, Université de Lomé-Togo,

doi: 10.19044/esj.2017.v13n27p251 URL:http://dx.doi.org/10.19044/esj.2017.v13n27p251

Abstract

Introduction: Osteoarticular infections remain public health problems in Africa. We aim at determining the clinical forms, topographic and etiological osteoarticular infections in a rheumatology unit of northern Togo. Methods: We conducted a cross-sectional study from April 2012 to March 2015 on inpatient records having suffered from musculoskeletal infection. Results: Of the 1813 patients admitted to the department in three years, $86(4.74 \%)$ suffered from musculoskeletal infection. Of them, 36 $(41.86 \%)$ were men and $50(58.1 \% 4)$ were women, with a sex ratio $(\mathrm{M} / \mathrm{F})$ of 0.72 . The mean age of the patients was 45 years. The mean duration of disease progression was 3.5 months. The different clinical forms observed were: spondylitis (47 patients, 54.65\%), infectious arthritis (31 cases, $36.05 \%$ ) and osteomyelitis (eight cases; 9.30\%). The infection was likely 
tuberculous in 53 patients (61.63\%), including 44 cases of Pott's disease. A banal germ was mentioned in the 33 others patients $(38.37 \%)$. In four cases, the germ was isolated: Staphylococcus aureus (three cases) and Staphylococcus epidermidis (one case). The joints most affected by the infection were the hip (nine patients) and the knee (eight patients). Infection was multifocal in 14 cases $(16.27 \%)$. The main risk factors for the infection identified were: promiscuity and poor hygiene $(59.30 \%)$, alcoholism $(26.74 \%)$ and retroviral infection $(12.79 \%)$. Conclusion: This study and joint infections are a common reason for rheumatology consultation in northern Togo with a significant share of multifocal forms.

Keywords: Epidemiology, infection, spondylitis, black Africa

\section{Résumé :}

Introduction : Les infections ostéoarticulaires demeurent un problème de santé publique en Afrique. Notre étude a eu pour objectif de déterminer les formes cliniques, topographiques et étiologiques des infections ostéoarticulaires dans un service de rhumatologie du nord Togo. Méthodes : Nous avons mené une étude transversale d'avril 2012 à mars 2015 sur les dossiers de malades ayant souffert d'une infection ostéoarticulaire. Résultats : Des 1813 patients admis dans le service en trois ans, $86(4,74 \%)$ d'entre eux souffraient d'une infection ostéoarticulaire. Ils se répartissaient en 36 hommes $(41,86 \%)$ et 50 femmes $(58,14 \%)$, soit un sex ratio $(\mathrm{H} / \mathrm{F})$ de 0,72 . L'âge moyen des patients était de 45 ans. La durée moyenne d'évolution de la maladie était de 3,5 mois. Les différentes formes cliniques observées étaient: la spondylodiscite (47 patients; 54,65\%), l'arthrite infectieuse (31 cas ; 36,05\%) et l'ostéomyélite (huit cas ; 9,30\%). L'infection était d'origine tuberculeuse probable chez 53 patients $(61,63 \%)$ dont 44 cas de mal de Pott. Un germe banal a été évoqué chez les 33 autres patients $(38,37 \%)$. Dans quatre cas, le germe a été isolé : Staphylocoque aureus (trois cas) et Staphylocoque épidermidis (un cas). Les articulations les plus touchées par l'infection était la coxo-fémorale (neuf patients) et le genou (huit patients). L'infection était multifocale dans 14 cas (16,27\%). Les principaux facteurs de risque de l'infection identifiés étaient : la promiscuité et l'insuffisance d'hygiène $(59,30 \%)$, l'éthylisme $(26,74 \%)$ et l'infection rétrovirale $(12,79 \%)$. Conclusion : Il ressort de cette étude que les infections ostéoarticulaires restent un motif fréquent de consultation rhumatologique au nord du Togo avec une part importante des formes multifocales.

Mots clés : Epidémiologie, infection, spondylodiscite, Afrique noire 


\section{Introduction}

Les infections ostéoarticulaires demeurent un problème de santé publique en Afrique comme le démontrent de nombreuses études (Jeandel \& al, 1991 ; N’tsiba \& al, 2006 ; Ouedraogo \& al, 2014 ; Nzenze \& al, 2001). Elles sont fréquentes à tous les âges dans cette partie du monde (Lavy, 2007; Kaabach \& al, 2002; Diomande \& al, 2015). Grâce aux études menées pour la plupart en milieu hospitalier, le rôle majeur joué par les agents infectieux dans le développement des affections rhumatismales a été mis en évidence. Cependant, aucune étude n'a été menée sur les infections ostéoarticulaires au nord du Togo. Ainsi, cette étude a eu pour but de déterminer la fréquence, le profil sémiologique et les formes étiologiques des infections ostéoarticulaires au cours d'une consultation rhumatologique dans cette partie d'Afrique.

\section{Méthodes}

Il s'est agi d'une étude transversale menée sur les dossiers de patients ayant consulté dans le service de rhumatologie du CHU de Kara $(420 \mathrm{~km}$ au nord de Lomé la capitale) d'avril 2012 à mars 2015. Etaient inclus dans l'étude tous les patients ayant souffert d'une infection ostéoarticulaire. Les données démographiques, cliniques et paracliniques de ces patients ont été recueillies sur une fiche d'enquête à partir de leurs dossiers. Le diagnostic positif de l'infection ostéoarticulaire était essentiellement radioclinique. Le diagnostic étiologique a reposé sur des arguments cliniques, biologiques et épidémiologiques. En raison de l'insuffisance de plateau technique, aucun patient n'a bénéficié d'une ponction biopsie discovertébrale.

\section{Résultats}

Sur les 1813 patients examinés durant la période d'étude, $86(4,74 \%)$ d'entre eux souffraient d'une infection ostéoarticulaire. Ils se répartissaient en $36(41,86 \%)$ hommes et $50(58,14 \%)$ femmes soit une sex ratio $(\mathrm{H} / \mathrm{F}) \mathrm{de}$ 0,72 . L'âge moyen de nos patients au diagnostic était de 45 ans (extrêmes : quatre ans et 80 ans). La durée moyenne d'évolution de la maladie était de 3,5 mois (extrêmes: quatre jours et 48 mois). Le mode d'installation de la maladie était brutal chez 40 patients $(46,51 \%)$ et progressif chez les 46 autres $(53,49 \%)$. L'horaire de la douleur était inflammatoire dans 41 cas $(47,67 \%)$, mixte dans 10 cas $(11,63 \%)$ et mécanique chez les 35 autres $(40,70 \%)$ cas. L'était général était altéré chez 63 patients $(73,25 \%)$ et une fièvre était présente chez 24 patients $(27,90 \%)$. Les formes cliniques d'infection ostéoarticulaire retrouvées étaient : les spondylodiscites infectieuses (47 cas : $54,65 \%$ ), les arthrites infectieuses (31 cas : 36,05\%) et les ostéomyélites (huit cas : 9,30\%). Une porte d'entrée infectieuse a été retrouvée chez 22 patients $(25,58 \%)$. Elle était cutanée dans 18 cas et digestif, gynécologique, pulmonaire, et urinaire dans un cas respectivement. La vitesse de 
sédimentation moyenne était de $66 \mathrm{~mm}$ à la première heure (extrêmes : $4 \mathrm{~mm}$ et $135 \mathrm{~mm}$ ). Un germe a été isolé chez quatre patients (4,65\%). Il s'agissait du Staphylocoque aureus dans trois cas et du Staphylocoque épidermis dans un cas. Chez 29 patients, l'infection était à germe banal probable. Le bacille de Koch (BK) a été suspecté chez 53 patients $(61,63 \%)$. L'atteinte tuberculeuse était associée à une pneumopathie chez sept patients. Les spondylodiscites étaient d'origine tuberculeuse probable dans 44 cas $(93,62 \%)$ et à germe banal probable dans les trois autres cas $(6,38 \%)$. Un abcès froid paravertébral était présent chez deux patients. L'atteinte rachidienne était bifocale chez cinq patients et multifocale chez deux patients. Les autres localisations du BK étaient : la coxofémorale (cinq cas), le genou (un cas) et une polysérite (polyartrhite touchant le tarse droit, le coude gauche et le poignet gauche, pleurésie et ascite) chez une patiente immunodéprimée.

La coxofémorale était l'articulation la plus touchée parmi les 31 cas d'arthrite infectieuse (tableau 1). L'atteinte infectieuse touchait au moins deux articulations chez quatre patients.

Tableau 1 : Répartition des patients selon l'articulation touchée par l'infection

\section{Nombre}

\begin{tabular}{cc}
\hline Coxofémorale & 9 \\
Genou & 8 \\
Epaule & 5 \\
Tarse & 4 \\
Coude & 3 \\
Main & 2 \\
\hline Total & 31
\end{tabular}

L'ostéomyélite infectieuse a affecté sept patients dont trois localisations au fémur, deux à l'humérus, une au tibia et une à l'aile ischiopubienne. L'atteinte était multifocale chez trois patients. Les mauvaises conditions d'hygiène et le sous développement étaient le facteur de risque le plus retrouvé (tableau 2).

Tableau 2 : Principaux facteurs de risque de l'infection retrouvés

\begin{tabular}{cc}
\hline & Nombre $(\%)$ \\
\hline Insuffisance d'hygiène et promiscuité & $51(59,30)$ \\
Ethylisme & $23(26,74)$ \\
Infection par le VIH & $11(12,79)$ \\
Hémoglobinopathie & $4(04,65)$ \\
Diabète & $3(03,48)$ \\
\hline
\end{tabular}




\section{Discussion}

Les infections ostéoarticulaires représentaient 4,74\% des motifs de consultation rhumatologique au CHU de Kara. En dépit des insuffisances (recrutement hospitalier, étroitesse du plateau technique), cette étude témoigne de la place prépondérante qu'occupent les infections ostéoarticulaires en pratique rhumatologique à Kara.

L'âge moyen de nos patients était de 45 ans, comparable à celui d'autres patients ouest-africains (Ouedraogo \& al, 2014; Houzou \& al, 2013 ; Eti \& al, 2000 ; Ogunlusi, 2006). Les caractéristiques démographiques et sémiologiques sont similaires à celles notées par d'autres auteurs africains (N'tsiba \& al, 2006; Oniankitan \& al, 2011; Mue \& al; 2013). L'atteinte multifocale n'est pas rare (N'tsiba \& al, 2006; Mue \& al; 2013). Elle pourrait s'expliquer par le retard diagnostique dû d'une part à l'éloignement des centres hospitaliers et d'autre part au recours fréquent aux tradipraticiens. Les spondylodiscites infectieuses sont dominées par le mal de Pott, confirmant la persistance de l'endémicité du BK en Afrique (Ouedraogo \& al, 2014 ; Maftah \& al, 2001 ; Badaoui \& al, 2014). Le fémur et l'humérus étaient les os les plus touchés par l'ostéomyélite infectieuse, confirmant les données de la littérature (Mayikoua \& al, 1992 ; Diémé \& al, 2014). Les arthrites infectieuses quant à elles siégeaient plus à la coxofémorale et au genou (Oniankitan \& al, 2011; N'tsiba \& al, 2004 ; Le Dantec \& al, 1999). Le staphylocoque aureus était le germe le plus souvent isolé comme dans la plupart des études menées dans le monde (N'tsiba \& al, 2006; Khan \& al, 2013; Eder \& al, 2005 ; Favero \& al, 2008). Bien que le germe n'ait été isolé que dans une faible proportion, il n'existe cependant pas de différences entre les caractéristiques sémiologiques des infections ostéoarticulaires à culture positive ou non (Madruga \& al, 2014). Contrairement à l'occident où les causes iatrogènes, les rhumatismes inflammatoires chroniques et les antécédents de prothèse sont les principaux facteurs de risque associés aux infections ostéoarticulaires (Favero \& al, 2008), l'insuffisance d'hygiène, l'éthylisme et l'infection par le VIH semblent en être les premières causes en Afrique (N'tsiba \& al, 2006 ; Eti \& al, 2000; Ogunlusi, 2006; Zomalheto \& al, 2010). Ceci devrait motiver l'intensification de la lutte contre le sous développement dans notre milieu.

\section{Conclusion}

Cette étude atteste de la fréquence encore élevée des infections ostéoarticulaires au Togo. Il n'y a pas de particularité sémiologique avec les études antérieures. Cependant, un affinement des moyens diagnostiques, permettrait de mieux cerner les germes responsables de ces infections. 


\section{References:}

1. Jeandel, P., Chouc, PY., \& Laroche, R. (1991). Rhumatologie en Afrique Noire: certitudes, perspectives et inconnues. Med Afr Noire ; 38 (1) : 53-61.

2. N'tsiba, H., Makosso, E., Ngandeu-Singwé, M., Yala, F. (2006). Les arthrites septiques en zone tropicale. A propos de 176 cas observés à Brazzaville. Mali Med; 21 : 49-53.

3. Ouedraogo, DD., N'tsiba, H., Tiendrébégo Zabsonré, J., Tieno, H., Bokossa, LI., Kaboré, F., \& Drabo, J. (2014). Clinical spectrum of rheumatologic diseases in a departement of rheumatology in Ouagadougou (Burkina Faso). Clin Rheumatol; 33 (3) : 385-9.

4. Nzenze, JR., Belembaogo, E., Magne, C., Sanou, AS., Coniquet, S., Moussavou- Kombila, JR., \& Boguikouma, JB. (2001). Panorama des arthropathies inflammatoires à Libreville. Analyse de 57 observations. Med Afr Noire ; 48 (10) : 399-402.

5. Lavy, CBD. (2007). Septic arthritis in western and sub-Saharian African children- a review. Int Orthop. 31:137-44.

6. Kaabach, O., Nessib, N., Thabet, L., Kechrid, A., \& Ben Gachem, M. (2002). Epidemiology of osteomyelitis and arthritis in infants. Tunis Med; 80:329-33.

7. Diomande, M., Eti, E., Ouali, B., Kouakou, ESCL., Yaconon, MO., Djaha, JM., Gbané- Koné, M., \& Kouakou, NM. (2015). Profil des affections ostéoarticulaires des sujets âgés noirs africains: à propos de 157 cas vus à Abidjan. Tunis Med ; 93:312-5.

8. Houzou, P., Oniankitan, O., Kakpovi, K., Koffi-Tessio, VES., Tagbor, KC., Fianyo, E. \& Mijiyawa, M. (2013). Profil des affections rhumatismales chez 13517 patients ouest africains. Tunis Med 2013;91:16-20.

9. Eti, E., Daboiko, JC., Debauly, S., Ouali, B., Ouattara, B., Yao, N., Gabla, A. \& Kouakou, NM. (2000). Arthrites à pyogènes des members au CHU de Cocody: A propos de 79 cas. Rhumatologie; 52:18- 21.

10. Ogunlusi, JD. (2006). Septic arthritis in a Nigerian tertiary hospital. Lowa Orthop J; 26: 45-7.

11. Oniankitan, O., Bagayogo, Y., Fianyo, E., Koffi-Tessio, V., Kakpovi, K., Tagbor, KC., Houzou P., \& Mijiyawa, M. (2011). Arthrites infectieuses en consultation rhumatologique à Lomé (Togo). Med Trop; 71:61-2.

12. Mue, DD., Salihu, MN., Awonusi, FO., Yongu, WT., Kortor, JN., \& Elachi, IC. (2013). The epidemiology and outcome of acute septic arthritis: A hospital based study. J West Afr Coll Surg ; 3:40-52. 
13. Maftah, M., Lmejjat, M., Mansouri, A., El Abbadi, N., \& Bellakhdah, F. (2001). Mal de Pott. A propos de 320 cas. Med Maghreb;90:19-22.

14. Badaoui, L., Dabo, G., Sodqi, M., Marih, L., Chakib, A., \& Marhoum, KEF. (2014). Spondylodiscites infectieuses: Aspects épidémio-cliniques paracliniques, thérapeutiques et évolutifs. Rev Mali Infect Microbiol; 3:29-33.

15. Mayikoua, A., Pena-Pitra, B., Ondzoto, JM., \& Kaya, JM. (1992). Ostéomyélites de 1'adulte: à propos de 25 cas. Med Afr Noire; 39:749-51.

16. Diémé, C., Sarr, L., Guèye, AB., Coulibaly, NF., Sane, A., Ndiaye, A., \& Sèye, S. (2014). Therapeutic aspects of bone infections and management challenges. Open Journal of orthopedics; 4:21-6.

17. N'tsiba, H., Bazébissa, R., Lamini, N., \& Yala, E. (2004). Knee septic arthritis: 100 cases report in intertropical zone. Bull Soc Pathol Exot; 97:244-6.

18. Le Dantec, L., Maury, F., Flipo, RM., Laskri, S., Cortet, B., Duguesnoy, B. \& Delcambre, B. (1996). Rev Rhum [Eng Ed ];63:103-10.

19. Khan, FY., Abu-Khattab, M., Baagar, K., Mohamed, SF., Elgendy, I., Anand, D., Malallah, H. \& Sanjay D. (2013). Characteristics of patients with definite septic arthritis at Hamad General Hospital, Quatar: a hospital based study from 2006 to 2011. Clin Rheumatol; 32:969-73.

20. Eder, L., Zisman, D., Rozenbaum, M., \& Rosner, I. (2005). Clinical features and aetiology of septic arthritis in northen Israel. Rheumatology (Oxford); 44:1559-63.

21. Favero, M., Schiavon, F., Riato, L., Carraro, V., \& Punzi L. (2008). Septic arthritis: a 12 years retrospective study in a rheumatological University clinis. Rheumatismo; 60: 260-7.

22. Madruga Dias, DJ., Costa, MM., Pereira da Silva, JA., \& Viana de Queiroz, M. '2014). Septic arthritis: patients with or without isolated infectious agents have similar charactéristics. Infection; 42:385-91.

23. Zomalheto, Z., Avimadje, M., Gounongbe, M., \& Dossou-Yovo, H. (2010). Facteurs liés à l'atteinte discovertébrale de la tuberculose à Cotonou. Le Bénin Médical;44: 30-32. 